fasting glucose, insulin, sex-steroids and sex hormone binding globulin from a morning blood sample, and muscle and fat mass from a dual-energy x-ray absorptiometry scan. Lifestyle was obtained from a questionnaire. We used multivariable linear regression to assess adjusted associations.

Results Free and bio-available testosterone were positively associated with muscle mass, as was physical activity. Muscle mass was associated with lower glucose $(-0.04 \mathrm{mmol} / \mathrm{l}, 95 \%$ CI -0.08 to -0.01 per $\mathrm{kg}$ muscle mass) and lower insulin $(-0.94 \mathrm{uU} / \mathrm{ml}, 95 \%$ CI -1.75 to -0.14 ); adjusted for sex and fat mass.

Conclusions Environmentally driven muscle mass acquisition at puberty could potentially be an additional factor influencing diabetes, of which further investigation is warranted.

\section{SP1-35 PREDICTORS OF CHANGE IN JOINT FUNCTION AFTER HIP OR WRIST FRACTURES DUE TO FALLS IN ELDERLY PEOPLE}

doi:10.1136/jech.2011.142976n.12

\begin{abstract}
IJ M Quintana, ${ }^{* 2}$ A Bilbao, ${ }^{1} S$ Garcia, ${ }^{1} \mathrm{C}$ Las Hayas, ${ }^{1} \mathrm{M}$ Orive, ${ }^{1} \mathrm{I}$ Barrio, ${ }^{3} \mathrm{G}$ Navarro. ${ }^{1}$ Hospital Galdakao-Usansolo, Galdakao, Bizkaia, Spain; ${ }^{2}$ Bioef, Sondika, Bizkaia, Spain; ${ }^{3}$ Corporació Sanitaria Parc Taulli, Sabadell, Barcelona, Spain
\end{abstract}

Introduction Falls with hip or wrist fractures in elderly people are common situations. The goal of this study was to determine the joint function and which clinical and social factors predict change after those fractures, measured by the short-WOMAC or QuickDASH questionnaires.

Methods Patients older than 65 years who attended the emergency room (ER) of seven acute hospitals with a hip or wrist fracture due to a fortuity fall were recruited. Patients fulfilled the QuickDASH or short WOMAC specific questionnaires at the time of the fall, as how they were before the fall, and 6 months later, and some other questions on sociodemographic issues. Clinical parameters from the ER and admission to the hospital were also recorded. Univariate and multivariate regression analysis were performed, considering the changes on the QuickDASH or short WOMAC specific questionnaires as dependent variables.

Results Preliminary analysis of our data (recruitment: 343 hip; 412 wrist fracture patients) showed an important decline in both specific questionnaires (hip-limitation domain of short-WOMAC-26.2; wrist-QuickDASH:20.3) at 6 months after the fracture. Older patients and lower socioeconomic status were those with greater worsening on WOMAC limitation domain on hip fracture, after adjusting by baseline scores. On wrist fractures, patients receiving rehabilitation and those who did not receive social support had greater worsening on QuickDASH scores.

Conclusion Specific changes in the function of the joint affected were determined by the socioeconomic level on hip fractures while in wrist fractures the presence of social support provided benefit for these patients.

\section{SP1-36 MORTALITY RATES OF PROSTATE CANCER AND DIETARY AND AGRICULTURAL VARIABLES: AN ECOLOGICAL STUDY IN BRAZIL}

doi:10.1136/jech.2011.142976n.13

${ }^{1} \mathrm{~L} \mathrm{~L} \mathrm{Luz,}$, 2,3 J F Santos-Silva, ${ }^{1} \mathrm{~L}$ M Santiago, ${ }^{1} \mathrm{I}$ E Mattos. ${ }^{1}$ Oswaldo Cruz Foundation, National School of Public Health, Rio de Janeiro, Brazil; ${ }^{2}$ Mato Grosso do Sul State Health Secretariat, Campo Grande, Mato Grosso do Sul, Brazil; ${ }^{3}$ Federal University of Mato Grosso do Sul State, Campo Grande, Mato Grosso do Sul, Brazil

Introduction Mortality rates of prostate cancer show regional variations in Brazil. The heterogeneous dietary profile and the distribution of agricultural practices could, at least partially, explain the observed patterns. This ecological study aimed to identify associations between selected dietary, agricultural variables and mortality rates for prostate cancer in men aged 60 or more in selected Brazilian States.

Methods States selected for study were the main agricultural producers in Brazil. Dietary (characterised as per capita kcal/day consumption of food groups) and agricultural variables were selected in the literature. Agricultural data were obtained from the National Agricultural Census and dietary data from the National Survey by Household Sampling. Multiple linear regression were used to analyse the correlations between mortality rates and the selected variables.

Results Age-adjusted mortality rates varied from 112.79 to 174.92 per 100000 . The final multivariate model was capable of explaining $99.9 \%$ of the variation in mortality rates. Number of agricultural establishments, hectares planted with permanent crops, and hectares planted with temporary crops showed positive associations with mortality rates, as well as vegetable consumption, percentage of population aged 60 or more, and percentage of population having had medical consultation in the last 12 months. Negative associations were observed for total calories, consumption of oils and fats and percentage of population with health plan coverage.

Conclusions These results suggest that differences in long-term dietary habits and exposures to agricultural hazards could influence patterns of prostate cancer among Brazilian elders. Further epidemiologic studies are needed to clarify these possible associations.

\section{SP1-37 THE USE OF THE ABBREVIATED COMPREHENSIVE GERIATRIC ASSESSMENT (ACGA) AS A SCREENING INSTRUMENT IN OLDER INDIVIDUAL LIVING IN LONG-STAY INSTITUTIONS IN BRAZIL}

doi:10.1136/jech.2011.142976n.14

L L Luz, ${ }^{*}$ L M Santiago, I E Mattos. Oswaldo Cruz Foundation, National School of Public Health, Rio de Janeiro, Brazil

Introduction The Comprehensive Geriatric Assessment (CGA) is used in the care of older person for performing a multidimensional evaluation. However, CGA is a time-consuming assessment and abbreviated screening instruments have been proposed. The purpose of this study was to analyse the performance of the Abbreviated Comprehensive Geriatric Assessment (aCGA) in elders living in long-stay institutions in Brazil.

Methods This is a study with elderly residents of long-stay institutions in four Brazilian cities. The assessment of functional, emotional and cognitive domains was performed with the instruments: Activities of Daily Living (ADL); Instrumental Activities of Daily Living (IADL); Geriatric Depression scale (GDS); Mini-Mental State Examination (MMSE) and with the aCGA. Each domain was scored separately with both instruments and individuals classified accordingly. Pearson's correlation coefficients and positive predictive values (PPV) were used to compare the abbreviated version with the full instruments.

Results These are preliminary results for 340 elders. Mean age was 75.5 years. The prevalence of dependence in $\mathrm{ADL}(65.0 \%$ vs $40.0 \%)$ and in IADL (72.9\% vs $58.5 \%)$ and of cognitive impairment $(84.9 \%$ vs $65.8 \%$ ) were higher when identified through aCGA, than with the full instrument. However, depression was less detected with the aCGA (27.6\% vs $31.5 \%)$. Correlations between abbreviated and full instruments ranged from 0.80 to $0.98(p<0.001)$. The highest PPV of the aCGA was observed for depression (86.9\%) and the lowest for $\mathrm{ADL}(58.4 \%)$

Conclusions In general, the aCGA performed well as a screening instrument in this setting. However, caution must be exercised in relation to the identification of depression. 